\title{
Effects of Methylated Xanthines on the Neuronally Isolated Cerebral Cortex
}

\author{
Ajit Maiti and Edward F. Domino ${ }^{1}$ \\ Department of Pharmacology, University of Michigan, Ann Arbor, Michigan
}

Received July 28, 1960

\begin{abstract}
Although caffeine has long been considered to be a cerebral cortical stimulant, relatively little is known of its sites and mechanism of action in the cerebral cortex. Therefore, the effects of the methylated xanthines, caffeine, theophylline, and theobromine have been studied on the evoked electrical afterdischarye of the neuronally isolated cerebral cortex preparation of dogs. Electrical stimulation of the neuronally isolated island of suprasylvian gyrus with an intact pial circulation in postanesthetized and immobilized dog preparations elicited a characteristic reproducible spikelike discharge limited within the island. The duration of such afterdischarge was measured before and after the administration of various drugs. Among the methylated xanthines, caffeine in doses as low as $0.25 \mathrm{mg} / \mathrm{kg}$ was the most potent in prolonging cortical afterdischarge. Theophylline and theobromine appeared much less potent. The dose-effect relationship with different doses of the methylated xanthines was determined. It was found that large doses of theophylline and theobromine clearly reduced the duration of cortical afterdischarge. Repeated administration of caffeine appeared to cause no further or even less prolongation of afterdischarge than that produced by the first dose suggesting that tachyphylaxis occurred.
\end{abstract}

\section{Introduction}

Caffeine is widely used as a psychic stimulant, especially as a natural constituent of several beverages such as coffee, tea, and maite. In spite of its widespread use since antiquity, relatively little is known of its sites and mechanism of action. Caffeine generally is assumed to be a cerebral cortical stimulant. Berger (1) observed that following the subcutaneous injection of caffeine sodium salicylate into human subjects, the

1 This research was supported in part by Grant MY-2653, National Institute of Mental Health, USPHS. A preliminary communication has been presented before the American Society for Pharmacology and Experimental Therapeutics (Federation Proc. 19: 269, 1960). Dr. Maiti has been supported by the International Cooperation Administration under the Visiting Research Scientists Program administered by the National Academy of Sciences of the United States of America. 
fast activity (his "beta waves," $60 \mathrm{cps}$ ) greatly increased in voltage and amount. He considered these effects to be related to the psychic stimulation. Gibbs and Maltby (8) reported that caffeine causes a shift in the human electroencephalogram frequency-spectrum toward the faster waves. Both an increase in frequency and voltage of the alpha rhythm were noted. Swank and Foley (12) reported that caffeine and theophylline ethylenediamine differed markedly from pentylenetetrazole and picrotoxin in having no marked effect upon the EEG of dogs in doses which were definitely analeptic in the therapy of barbiturate coma. Caffeine increases the oxygen consumption of in vitro frog brain slices (2). When it is added to a saline solution bathing the isolated frog brain it produces large electrical slow wave potentials. DC potentials may be recorded with electrodes on the pial and ventricular surfaces of the frog brain (9). Libet and Gerard suggested that caffeine may alter cell membrane impedance.

The purpose of this investigation was to study the effects of caffeine and related methylated xanthines on the neuronally isolated ccrebral cortex of dogs. Cortical afterdischarge evoked by repetitive electrical stimulation of the isolated island was used as an index of activity which could be quantified.

\section{Methods}

Experiments were performed on mongrel dogs of both sexes weighing from 6 to $12 \mathrm{~kg}$. Surgery was performed on animals anesthetized with halothane $e^{2}$ in a 2 to 3 per cent concentration in oxygen administered via a Fluotec vaporizer. The femoral artery was cannulated for blood pressure recording. A Statham P23 strain gauge connected to a Grass Balance-Demodulator recorded blood pressure on one channel of the Grass electroencephalograph. In addition, the blood pressure was recorded directly using a mercury manometer. The parietal cortex was exposed by means of a trephine and rongeurs. After the dura was removed, a portion of suprasylvian gyrus approximately $15 \mathrm{~mm} \times 10 \mathrm{~mm}$ and $3 \mathrm{~mm}$ deep was isolated neuronally with a relatively intact pial circulation. The techniques used were similar to those of Burns, as modified by Rech and Domino (11).

After completion of surgery the anesthesia was discontinued and the animal placed on artificial respiration. The subject was kept immobilized by the administration of decamethonium, $0.5 \mathrm{mg} / \mathrm{kg}$ given intravenously as necessary. In order to prevent pain all wound sites were infiltrated

2 Kindly supplied by Dr. J. B. Jewell, Ayerst Labs. New York 16, N.Y. 
with 2 per cent procaine. For recording the electrical activity from the cerebral cortex bipolar cotton wick tipped silver electrodes soaked in saline were placed approximately $1 \mathrm{~cm}$ apart along the long axis of the gyrus in the isolated and intact cortex. Bipolar stimulating electrodes of similar design were placed in a line perpendicular to the recording electrodes. Usually about $1 \frac{1}{2}$ hours elapsed after the surgical operation before the onset of the experiment. Electrical afterdischarge was induced in the isolated area by stimulation with square wave pulses of $60 \mathrm{cps}$ for $2 \mathrm{sec}$. The pulse width was $1 \mathrm{msec}$ in duration. The threshold voltage for producing cortical afterdischarge in the isolated cortex varied between 2 and 8 volts, depending upon the animal. Occasionally monopolar recordings were used to determine the polarity of the induced afterdischarge. Under these circumstances a needle was inserted into a crushed region of the temporal muscle which served as the indifferent recording electrode. Considerable variation was observed in the duration of cortically evoked afterdischarge when threshold stimuli were used. Therefore submaximal stimuli were used to obtain cortical afterdischarge of approximately similar durations. Caffeine, theophylline, and theobromine as the base were prepared in either saline or water solution depending upon their solubility. In general, the $\mathrm{pH}$ of the solutions used was adjusted to approximately 6.4. The action of atropine, physostigmine, $d$-amphetamine, epinephrine, and oxygen were also studied in a few animals. All drugs were administered intravenously. The temperature of the exposed cortical areas was kept nearly constant at $37^{\circ} \mathrm{C}$. The area was kept moistened by frequent irrigation with warm 0.9 per cent saline solution. At the termination of about 20 per cent of the experiments the brains were prepared for histological study with semiserial sections to confirm the extent of the surgical isolation. In general it was observed that the isolation was complete except for small bridges in the molecular layer as described by Domino (6) for the cat.

\section{Results}

Physiological Characteristics of Induced Afterdischarge. The principal problem in the use of the in vivo isolated cerebral cortex preparation to study the action of drugs was the difficulty in obtaining suitable controls. A great deal of effort was necessary in order to obtain reproducible durations of evoked cortical afterdischarge in the isolated cerebral cortex. Although a thorough analysis of the parameters of stimulation was not undertaken, the effects of the time interval between electroshocks and 
the threshold voltage of stimulation were studied. At the beginning of the experiment, the threshold of afterdischarge in the isolated cortex was determined. The voltage of stimulation varied from 1.5 to 10 volts. Two to 8 volts applied for approximately $2 \mathrm{sec}$ were found optimal to produce submaximal afterdischarge. Increasing the voltage in general increased the duration of afterdischarge to a maximum of approximately 40 to 50 sec. The voltage was kept at the level found optimal for each particular animal in order to obtain reproducible afterdischarge of approximately 10 to $20 \mathrm{sec}$. Various time intervals of 1 to $10 \mathrm{~min}$ between each stimulus were used. The time interval was measured from the end of the afterdischarge in response to the previous shock to the next electrical stimulation. Five-minute intervals between stimuli were found to be suitable for approximately reproducible durations of cortical afterdischarge. The afterdischarge could be evoked at shorter intervals from 1 to 4 min but its duration was more variable. Frequently electrical afterdischarge was not discernible if the stimulus was applied within $30 \mathrm{sec}$ of the previous electroshock.

At the conclusion of a 2-sec electrical stimulus at threshold or above a train of high voltage (200 to $500 \mu \mathrm{v})$ multiphasic repetitive spikelike discharges of approximately 10 to $15 \mathrm{cps}$ or more appeared either immediately or after a very short latency ( $1 \mathrm{sec}$ or less) following the stimulus.

When the stimulus was applied to the isolated cerebral cortex the afterdischarge usually remained within the boundaries of the surgical isolation. Seldom did this activity spread to surrounding normal cortex. It was not always possible to explain the occasional spread of afterdischarge from isolated to normal cortex on the basis of inadequate isolation although this frequently appeared to be the case. When the cortical afterdischarge was initiated in the normal cortex frequently there was evidence of spread to the isolated region across the cut but this occurred usually much later than that in the intact area. These findings confirm the observations of Torres, Ziegler, and Wicoff (13). Minimal spontaneous electrical activity was observed from the isolated cortex. This was similar in character to that reported previously by Rech and Domino (11).

The character of the afterdischarge induced by electrical stimulation of the normal cortex differed from that evoked by stimulation of the isolated island. The former consisted generally in a series of repetitive spikes of 200 to $600 \mu \mathrm{v}$ gradually increasing in amplitude with a frequency of 10 to $20 \mathrm{cps}$. This activity ended abruptly with a short period of electrical silence. The afterdischarge in the isolated cortex consisted 
of a similar train of high voltage multiphasic repetitive discharges of 200 to $500 \mu \mathrm{v}$ and a frequency of 10 to $15 \mathrm{cps}$ which appeared immediately or after a brief period of build-up. The discharge pattern commonly persisted for 10 to $20 \mathrm{sec}$ or more, depending upon the voltage of stimulation and often became replaced by intermittent rhythmic activity following the afterdischarge. This rhythmic activity occurred at a rate of 2 to $5 \mathrm{cps}$ in which frequently a suppression burst pattern reappeared at the same frequency as before stimulation. Such activity was not observed in intact cortex. The isoelectric period frequently seen in normal cortex after a period of cortical afterdischarge was not as clearly discernible in the isolated cortex.

The cortical afterdischarge induced in the isolated preparation consisted of three characteristic patterns. The first of these included spikelike waves initially continuous becoming more segregated into groups separated by periods of relative electrical inactivity. A second pattern of rhythmic waves instead of intermittent trains of spikes was also observed. The frequency of these waves increased to a certain extent with time. This reached a maximum of approximately $10 \mathrm{cps}$. The third pattern of activity involved the amplitude of individual spikes which in creased at the beginning frequently as a staircase phenomenon and then remained at the same high level when the frequency increased. Under normal circumstances before isolation the same portion of suprasylvian gyrus which was stimulated at a threshold or above caused a marked spread of electrical afterdischarge into neighboring areas in all directions. After isolation the cortical afterdischarge usually remained within the island of isolation. Under these circumstances the threshold for eliciting cortical afterdischarge was elevated compared to that prior to the isolation.

Effects of Methylated Xanthines on Afterdischarge: Isolated Cortex. Intravenous administration of caffeine in doses of 0.25 to $1 \mathrm{mg}$ per kilogram enhanced the duration of cortical afterdischarge following supramaximal electroshocks. Frequently the duration of cortical afterdischarge of approximately 10 to $20 \mathrm{sec}$ was noticeably prolonged after a single dose of caffeine within 10 min after intravenous administration. The peak effect of prolongation of afterdischarge following caffeine usually occurred within 30 to 40 min after injection. The prolongation of cortical afterdischarge persisted for approximately 2 to 3 hours after which the duration of afterdischarge approached that of control. The effects of caffeine given intravenously on evoked afterdischarge in the isolated cortex are shown in Fig. 1. In panel A is illustrated a control afterdischarge elicited 

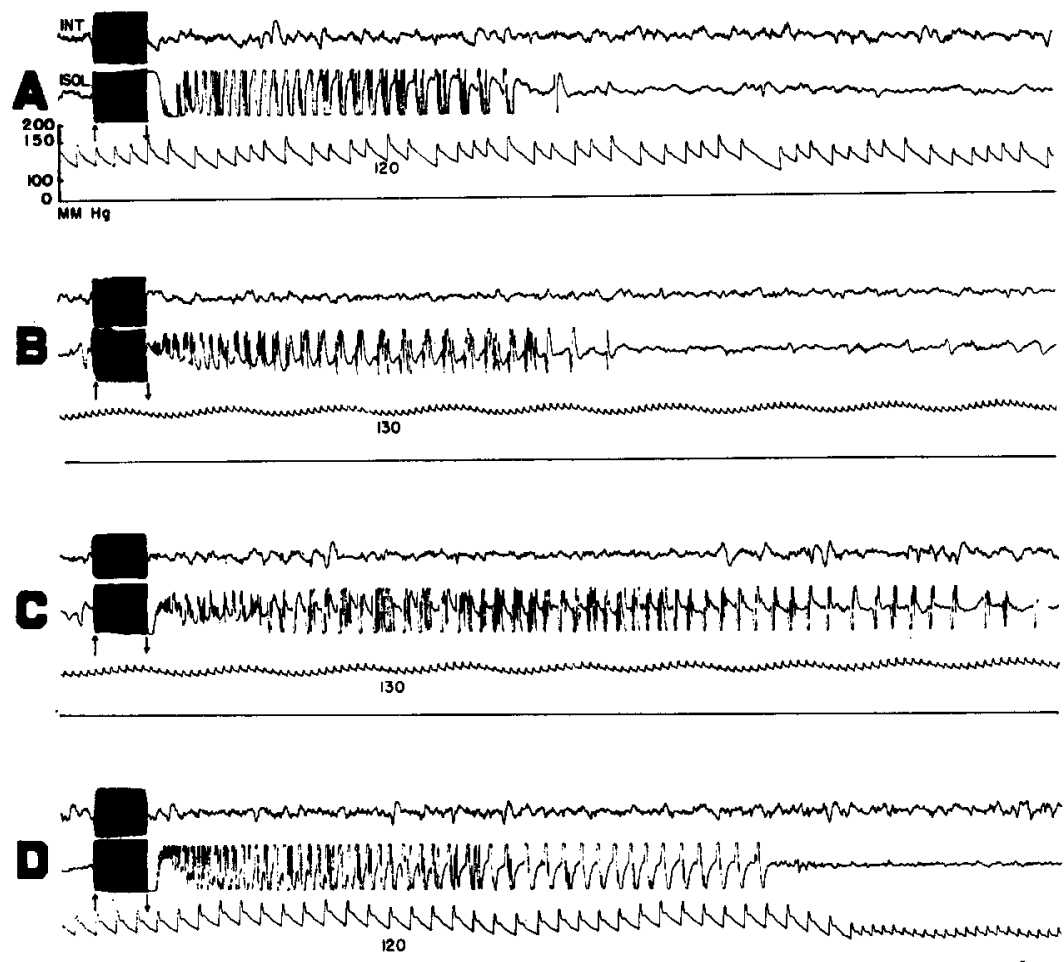

120

FIG. 1. EEG effects of caffeine on electrical afterdischarge in the dog isolated cerebral cortex. A, control spontaneous electrical activity and evoked cortical afterdischarge; $\mathrm{B}, 10 \mathrm{~min}$ after the injection of $0.5 \mathrm{mg} / \mathrm{kg}$ of caffeine base given intravenously; C, EEG activity $30 \mathrm{~min}$ after the administration of caffeine; D, partial recovery 2 hours after the administration of caffeine; amplitude, $100 \mu \mathrm{v}$; time, $2 \mathrm{sec}$. The symbols are: INT, bipolar recordings of EEG activity from the intact ectolateral gyrus bordering the portion of isolated suprasylvian cortex; ISOL, bipolar recordings of EEG activity from the isolated suprasylvian gyrus. The upward arrow illustrates the onset of the electrical stimulus applied to the isolated cerebral cortex and the downward arrow its cessation. The parameters of electrical stimulation were 60 -cycle square wave pulses of $1 \mathrm{msec}$ duration, 4-volts intensity for $2 \mathrm{sec}$. The interval between elicited afterdischarges was $5 \mathrm{~min}$. The femoral arterial blood pressure record in $\mathrm{mm} \mathrm{Hg}$ is illustrated as the third tracing of each panel. Inasmuch as the electrical system for recording blood pressure was extremely nonlinear the actual $\mathrm{mm} \mathrm{Hg}$ pressure as measured from the mercury manometer is listed below each blood pressure tracing. 
from stimulation of the island of isolated cortex with square wave pulses of 4-volts intensity. The upper tracing (INT) shows the spontaneous electrical activity of a portion of intact cortex while the middle tracing (ISOL) shows the spontaneous activity from the isolated area. Note the lower amplitude of spontaneous activity from the isolated area. The lower tracing of panel A illustrates the blood pressure record. This particular animal had a mean pressure of about $120 \mathrm{~mm} \mathrm{Hg}$. Immediately after electrical stimulation of the isolated cortex marked high voltage spikelike activity was elicited. No electrical seizurelike activity was observed in the surrounding normal cortex at this time. Following the electrical afterdischarge spontaneous activity was still observed in the isolated cortex. After a series of such control afterdischarges a single dose of caffeine $(0.5 \mathrm{mg} / \mathrm{kg})$ given intravenously prolonged the duration of cortical afterdischarge as can be observed in panel B, Fig. 1. The normal control duration of cortical afterdischarge of approximately $20 \mathrm{sec}$ was enhanced within $10 \mathrm{~min}$ as illustrated. The maximum prolongation of cortical afterdischarge by caffeine is shown in panel $\mathrm{C}$. This occurred usually within $30 \mathrm{~min}$ after injection. After several hours the duration of cortical afterdischarge decreased toward control levels. Approximately 2 hours after the intravenous administration of caffeine cortical afterdischarge was still prolonged as observed in panel D, Fig. 1. At this time the blood pressure tracing was not completely normal but alternated with bouts of normal heart rate activity and tachycardia. A tachycardia and a slight increase in blood pressure were frequently observed following the administration of caffeine. The effects of caffeine on the threshold of cortical afterdischarge was studied in four animals. Although a precise determination of the threshold was impossible to obtain because of the variability inherent in this preparation no marked changes induced by caffeine were noted.

Inasmuch as the duration of cortical afterdischarge also varies considerably in the control period it was felt important to evaluate these results statistically. In addition dose-response curves were obtained for caffeine, theophylline, and theobromine. The experimental design consisted of groups of approximately four dogs each administered randomly varying doses of caffeine, theophylline, theobromine, or 0.9 per cent saline. In Fig. 2 is illustrated a bar graph which summarizes some of the results obtained. The maximum duration of afterdischarge within 30 min was taken as the estimate of the drug effect. The solid black bars represent the mean control cortical afterdischarge of all animals before a given 
dose of each drug. The white bars represent the mean prolongation of cortical afterdischarge in four dogs per dose of caffeine. Similarly the slanted lined bars represent the mean prolongation of cortical afterdischarge after theophylline, and the dotted bars the effects of theobromine. The black vertical lines represent the standard deviation of the mean. It can be seen that there is considerable variation in the control mean amplitude of cortical afterdischarge. This varied approximately from $17 \pm 5.1 \mathrm{sec}$ to $23.6 \pm 4.4 \mathrm{sec}$. An attempt was made in each animal to obtain cortical afterdischarges of approximately $20 \mathrm{sec}$. The

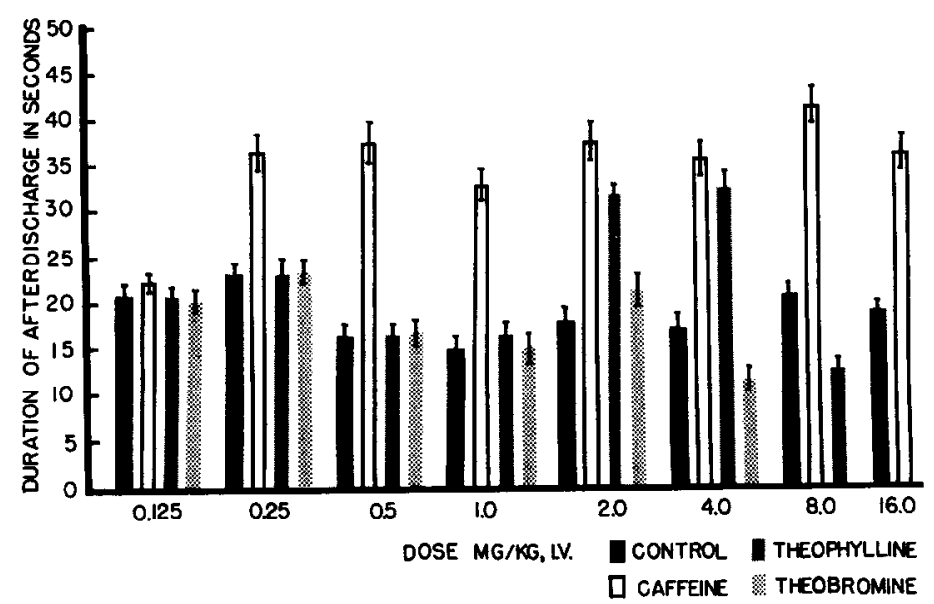

Fic. 2. The effects of the methylated xanthines on the duration of cortical afterdischarge expressed in seconds. Each of the appropriately diagrammed bars represents the drug administered intravenously as the base. The means are expressed for approximately a total of four different dogs per each dose of each drug. The control represents the pooled mean of all of these animals. The means are represented by the height of the individual bars and the standard error above and below the mean by the short vertical lines.

voltage was varied in each animal until responses of this duration were obtained. The administration of $0.25 \mathrm{mg} / \mathrm{kg}$ or more of caffeine intravenously markedly prolonged cortical afterdischarge. In all doses used the prolongation was statistically significant with a $P$ value of 0.001 or less, as determined by the "student" paired comparison " $t$ " test. After doses of 0.5 to $16.0 \mathrm{mg} / \mathrm{kg}$ the duration of cortical afterdischarge appeared to reach a maximum of approximately 33 to $42 \mathrm{sec}$. Theophylline in doses below $1 \mathrm{mg} / \mathrm{kg}$ given intravenously was ineffective in prolonging cortical afterdischarge. Doses of 2 and $4 \mathrm{mg} / \mathrm{kg}$ were significantly effec- 
tive in prolonging cortical afterdischarge. However, after a dose of 8 $\mathrm{mg} / \mathrm{kg}$ of theophylline the duration of cortical afterdischarge was reduced below control levels. Theobromine was especially difficult to study because of its insolubility. In doses of $1 \mathrm{mg} / \mathrm{kg}$ and less it had no effect on cortical afterdischarge. On the other hand $2 \mathrm{mg} / \mathrm{kg}$ of theobromine given intravenously did increase the mean duration of cortical afterdischarge. After $4 \mathrm{mg} / \mathrm{kg}$ of theobromine, which represented an extremely large volume of solution injected, theobromine produced a clear-cut depression of cortical afterdischarge. Doses above $4 \mathrm{mg} / \mathrm{kg}$ were not used because of the excessively large volumes of solution $(50 \mathrm{ml})$ that had to be injected.

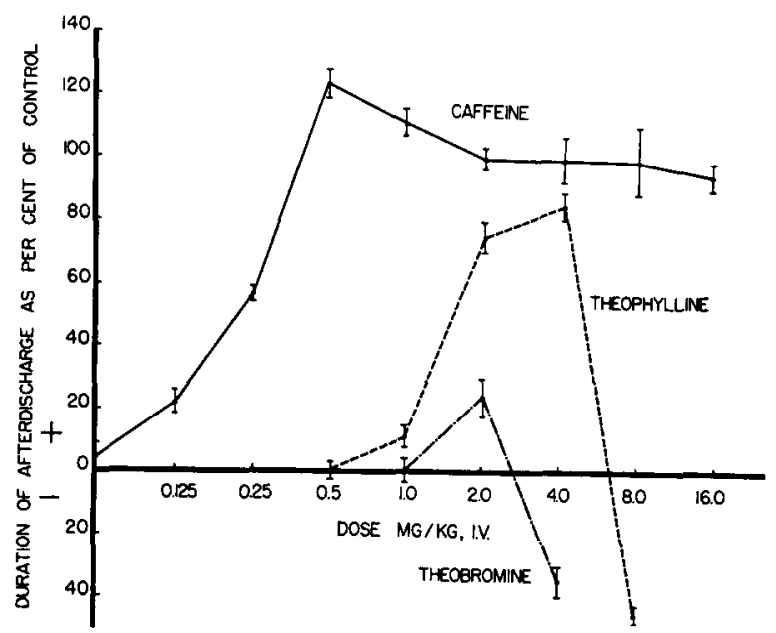

FIg. 3. The effects of the methylated xanthines on the duration of cortical afterdischarge expressed on a percentage basis. The data of Fig. 2, using the means of each animal's own control as 100 per cent, are plotted. The per cent prolongation above and below the control is expressed by the appropriate symbols + and - . The means and standard errors for each point represent four different animals.

The data illustrated in Fig. 2 on the basis of total duration of cortical afterdischarge expressed in seconds was reanalyzed and cxpressed as the per cent alteration of control. A summary of the effects of the methylated xanthines on the duration of cortical afterdischarge expressed as per cent of control is illustrated in Fig. 3. It can be seen that caffeine prolongs cortical afterdischarge to a maximum in a dose of about $0.5 \mathrm{mg} / \mathrm{kg}$. Larger doses up to $16 \mathrm{mg} / \mathrm{kg}$ were not more effective. Theophylline appears less effective than caffeine in prolonging cortical afterdischarge. 
Doses of $8 \mathrm{mg} / \mathrm{kg}$ produce some decrease in the duration of afterdischarge. Theobromine was least effective in prolonging cortical afterdischarge. It was found that the percentage prolongation of cortical afterdischarge varied considerably depending upon the duration of control. When the duration of afterdischarge was relatively short in the control period the percentage of prolongation produced by the methylated xanthines was much greater than when the duration of the controls was long. When control afterdischarges of approximately 30 to $40 \mathrm{sec}$ were obtained the percentage of prolongation was comparatively small, though in all cases there was some enhancement. Because of this, data expressed on a percentage basis tend to exaggerate differences perhaps more than when results are compared on the basis of simple prolongation of cortical afterdischarge expressed in seconds.

Repeated intravenơ injections of effective doses of caffeine in the order of 0.25 to $1 \mathrm{mg} / \mathrm{kg}$ given intravenously as well as effective doses of the other two methylated xanthines appeared to have much less or no effect on prolongation of cortical afterdischarge as was produced by the first dose. This was particularly evident in the case of caffeine. These effects were interpreted as representing tachyphylaxis. Two to 3 hours or more after the first dose of caffeine a second dose was again effective. It appeared that tachyphylactic responses were especially marked for the first few hours and became less obvious 3 to 4 hours after the first injection. Control experiments involving the injection of different volumes of 0.9 per cent saline were conducted in four dogs. No significant changes were noted. In these animals the mean control cortical afterdischarge \pm the standard deviation of the mean before saline was $20 \pm 6.81 \mathrm{sec}$ and after the administration of saline was $21 \pm 6 \mathrm{sec}$.

Effects of Methylated Xanthines on Afterdischarge: Intact Cortex. In six animals with the suprasylvian gyrus exposed, but not isolated, electrical afterdischarge was elicited with relatively low voltages (1-2 volts). In these animals caffeine in doses of $0.5 \mathrm{mg} / \mathrm{kg}$ given intravenously markedly prolonged cortical afterdischarge to $60 \mathrm{sec}$ or more. On a percentage basis cortical afterdischarge was increased to 300 or more per cent in contrast to about 120 to 140 per cent in the isolated cortex preparations.

No clear effects of the methylated xanthines were noted in the doses used on the spontaneous electrical activity of the normal as well as the isolated island of cortex.

Effects of Physostigmine and Atropine on Cortical Afterdischarge of 
Isolated Cortex. In a group of six animals physostigmine in doses of 50 to $200 \mu \mathrm{g} / \mathrm{kg}$ injected intravenously markedly prolonged the cortical afterdischarge in the isolated cortex within $10 \mathrm{~min}$. The peak effect usually occurred within $20 \mathrm{~min}$. This prolongation of afterdischarge persisted for about $30 \mathrm{~min}$ after injection and gradually subsided. In larger doses $(500 \mu \mathrm{g} / \mathrm{kg}$ ) physostigmine decreased the duration of cortical afterdischarge. Caffeine in optimal doses $(0.5 \mathrm{mg} / \mathrm{kg})$ caused a further increase in the duration of cortical afterdischarge after physostigmine even when there was marked shortening of the duration of afterdischarge by the latter. The percentage prolongation of the duration of cortical afterdischarge by caffeine $(0.5 \mathrm{mg} / \mathrm{kg})$ was always higher than that caused by optimal doses of physostigmine $(200 \mu \mathrm{g} / \mathrm{kg})$. Repeated injections of physostigmine $(200 \mu \mathrm{g} / \mathrm{kg})$ enhanced the duration of afterdischarge in most of the cases when the action of a previous dose was still evident. Thus no tachyphylaxis was demonstrated.

Atropine sulfate in doses of $1 \mathrm{mg} / \mathrm{kg}$, intravenously, did not have any clear effect on the duration of cortical afterdischarge induced in the isolated cortex nor did it influence the prolongation of afterdischarge caused by caffeine when injected after the caffeine. However, in atropine pretreated animals caffeine did not prolong the duration of cortical afterdischarge. In contrast, atropine injected either before or after physostigmine blocked the latter's augmentation of cortical afterdischarge.

Incidental Observations of Effects of Other Agents on Afterdischarge of Isolated Cortex. In a few animals the effects of $d$-amphetamine, epinephrine, glucose, and oxygen were studied on the duration of cortical afterdischarge. $d$-Amphetamine in doses of $0.5 \mathrm{mg} / \mathrm{kg}$ injected intravenously caused a marked increase in the blood pressure but there was no consistent increase in the duration of electrically induced cortical afterdischarge. Similarly epinephrine $(10 \mu \mathrm{g} / \mathrm{kg})$ given intravenously produced variable results in the duration of cortical afterdischarge. Glucose in high concentrations $(100 \mathrm{mg} / \mathrm{kg})$ injected intravenously was ineffective in prolonging the induced cortical afterdischarge. Oxygen insufflation through the respiratory pump caused a definite prolongation of cortical afterdischarge.

\section{Discussion}

It seems well established that the in vivo isolated cerebral cortex preparation appears to be suitable for the study of the cortical actions of various pharmacological agents. Such a preparation is relatively free of the modulating influences present in normal cortex. Investigations 
of the electrical properties of the isolated cortex and their modification by drugs has been performed by many (see 11). As demonstrated by Burns (3), electrical afterdischarge can be elicited in the isolated island of cerebral cortex. It has been postulated that the afterdischarge results from the excitation of reverberating circuits of cortical neurons (5). Although the evoked afterdischarges involving intrinsic cortical neuronal circuits are independent processes, it is obvious that these can easily be modified by subcortical or intracortical influences. The use of the in vivo isolated cortex preparation would keep such extrinsic influences to a minimum. Cortical afterdischarge has often been described as an extreme form of facilitation (4). If this is so, one can conclude that the methylated xanthines have a direct stimulating effect on the cerebral cortex. The role of cerebral vascular effects in this phenomenon are unknown. It is of course impossible to conclude that the effects of caffeine in prolonging cortical afterdischarge in the dog are necessarily related to the psychic phenomenon produced by caffeine in man. There is a considerable amount of pharmacological literature to support the notion that caffeine acts at many levels of the central nervous system of animals. Recently Funderburk and Woodcock (7) showed that caffeine prolonged cortical afterdischarge in cats with mesencephalic transections. Our own data would suggest that one site of action of caffeine in prolonging afterdischarge is the cortex itself. It is important to note that the prolongation of afterdischarge in dogs with an intact cortex was much greater than in the isolated preparation. The extent of local damage due to the surgical procedures or the role of ascending modulating influences in this phenomenon are unknown.

The analeptic or psychic stimulation produced by caffeine in man has been postulated to be due to a cortical site of action. The evidence offered in this investigation would only tend to support such a notion. Of special interest is that relatively small doses of caffeine (on a basis of milligram per kilogram) similar to those obtained in a cup of coffee in man produce clear cut prolongation of cortical afterdischarge in dogs.

The primary effect of caffeine was to prolong cortical afterdischarge and not to modify its threshold. Although caffeine only prolonged the duration of cortical afterdischarge, larger doses of theophylline and theobromine clearly reduced it. Perhaps if caffeine were given in very large doses a depressant effect might have also been observed. The interpretation of these effects of the methylated xanthines on cortical afterdischarge is unknown. The prolongation or shortening of cortical 
afterdischarge could be explained on the basis of inhibition or facilitation of a cortical inhibitory or facilitatory system. Only further research will provide the answers.

The methylated xanthines have been reported to have antiacetylcholinesterase properties (10). Physostigmine and atropine were administered in an attempt to determine whether these effects were related to the inhibition of acetylcholinestcrase within the ccrebral cortex. It was observed that low doses of physostigmine clearly prolonged cortical afterdischarge, while very large doses tended to reduce it. Of some interest was the fact that physostigmine did not prolong cortical afterdischarge to the extent produced by caffeine. Atropine abolished the action of physostigmine in prolonging cortical afterdischarge, but did not modify the prolongation of cortical afterdischarge by caffeine when it was given subsequently. When atropine was given as a pretreatment it did block the effects of physostigmine as well as caffeine. Although it is premature to postulate at this time the explanation of this very curious phenomenon, it seems that atropine pretreatment can prevent the molecules of caffeine from attaching to critical receptor sites. The administration of atropine after caffeine does not dislodge the caffeine-receptor combination. Obviously further research is necessary to confirm this unexpected finding.

\section{References}

1. Berger, H., Uber das Elektrenkephalogramm des Menschen, XIII. Arch. Psychiat. Nervenkrankh. 106: 577-584, 1937.

2. Brooks, V. B., R. E. Ransmeir, and R. W. Gerard, Action of anticholinesterases, drugs and intermediates on respiration and electrical activity of the isolated frog brain. Am. J. Physiol. 157: 299-316, 1949.

3. Burns, B. D., Some properties of isolated cerebral cortex in the unanesthetized cat. J. Physiol. London 112: 156-175, 1951.

4. Burns, B. D., "The Mammalian Cerebral Cortex." London, Edward Arnold Ltd., 1958.

5. Chanr, H-T., The evoked potentials. Handbook Physiol., Sect. I, Neurophysiol. 1: 305-312, 1959.

6. Domino, E. F., Pharmacological actions of a convulsant barbiturate II. Effects compared with pentobarbital on cerebral cortex and some brain stem systems of the cat. J. Pharmacol. Exptl. Therap. 119: 272-283, 1957.

7. Funderburk, W. H., and R. T. Woodcock, Comparative effects of analeptic drugs on the central nervous system. J. Pharmacol. Exptl. Therap. 119: 148, 1957.

8. GibBs, F. A., and G. L. MaLtby, Effect of electrical activity of the cortex of certain depressant and stimulant drugs-barbiturates, morphine, caffeine, benzedrine and adrenaline. J. Pharmacol. Exptl. Therap. 78: 1-10, 1943. 
9. Libet, B., and R. W. Gerard, Steady potential fields and neurone activity, $J$. Neurophysiol. 4: 438, 1941.

10. Nachmanson, D., and $H$. SchueEman, On the effect of drugs on cholinesterase. J. Biol. Chem. 159: 239-240, 1945.

11. RecH, R. H., and E. F. Domino, Effects of various drugs on activity of the neuronally isolated cerebral cortex. Exptl. Neurol. 2: 364-378, 1960.

12. Swank, R. L., and J. M. Foley, Respiratory, electroencephalographic and blood gas changes in progressive barbiturate narcosis in dogs. J. Pharmacol. Exptl. Therap. 92: 381-396, 1948.

13. Torres, F., K. ZIEgler, and H. S. WiCoff, Spontaneous and induced activity of the isolated cortex in cats. Electroencephalog. and Clin. Neurophysiol. 10: $190,1958$. 